\title{
Deep Brain Stimulation- A Novel Approach For Relieving Neurological Disorders: A Review
}

\author{
Archana Gautam ${ }^{1}$, Mansi Rai ${ }^{2}$, Rahul Srivastava ${ }^{3}$, Bhuvan jyoti ${ }^{4}$, Pankaj Kumar Priyadarshi ${ }^{5}$ \\ ${ }^{1}$ Senior Lecturer, Department of conservative and endodontics, Rama Dental College, Hospital \& Research Centre Kanpur \\ (U.P), ${ }^{2}$ Senior Lecturer, Department of conservative and endodontics, Rama Dental College, Hospital \& Research Centre \\ Kanpur (U.P), ${ }^{3}$ Reader, Department of Oral medicine and Radiology, Rama Dental College, Hospital \& Research Centre \\ Kanpur (U.P), ${ }^{4}$ Dental Surgeon and Consultant- Oral Medicine and Radiology, Department of Dental Surgery, Ranchi Institute \\ of Neuro-Psychiatry and Allied sciences, Ranchi, Jharkhand, India, ${ }^{5}$ Senior Lecturer, Department of conservative and \\ endodontics, Rama Dental College, Hospital \& Research Centre Kanpur (U.P)
}

Corresponding author: Archna Gautam, MDS, Senior Lecturer, (Conservative and Endodontics) Rama Dental College, Hospital \& Research Centre, Kanpur (U.P)., India

DOI: 10.21276/ijcmsr.2018.3.3.19

How to cite this article: Gautam A, Rai M, Srivastava R, Jyoti B, Priyadarshi PK. Deep brain stimulation- a novel approach for relieving neurological disorders: a review. International Journal of Contemporary Medicine Surgery and Radiology. 2018;3(3):C86-C92.

\section{A B S T R A C T}

Deep brain stimulation (DBS) is a neurosurgical procedure involving the implantation of a medical device called a neurostimulator (brain pacemaker) which transmits electrical impulses through embeded electrodes to specific sites of brain (brain nuclei) for the treatment of problem in movement and neuropsychiatric disorders. DBS in certain sites of brain has provided therapeutic benefits for disorders which are not treatable. This review highlights the principle mechanism of action of deep brain stimulation (DBS) advantages, limitations and it's amazing application in the management of various neurological disorders.

Key words: Deep Brain Stimulation, Neurological Disorders, Electrical Stimulation

\section{INTRODUCTION}

Electrical stimulation is a procedure which involves the introduction of a weak electrical current into the specific locations of the brain by using multiple microelectrodes. Short pulses of electrical currents are introduced intended to mimic natural flow of impulses through the neural pathways. ${ }^{1}$ In 1809, Luigi Ronaldo stimulated the cortical cortex of animals with the help of galvanic current. In 1874, Dr. Robert Bartholow was the first person to report the electrical stimulation findings of cerebral cortex in conscious human. In 1938, Ugo Cerletti introduced "Electric Shock" as therapeutic application of brain stimulation for the treatment of severe psychosis. Brain stimulation used as early as 1950 by placing temporary electrodes implanted into the brain regions for pain control. In early $19^{\text {th }}$ century, electrical stimulation was performed by Aldini, Luigi Galvani and professor of Physics at the University of Bologna on the exposed human cerebral cortex of recently decapitated prisoners. Aldini in 1804 suggested that cortical stimulation evoked horrible facial grimaces and it was concluded that electronic stimulation have therapeutic effects in cases of neuropsychiatric disorders. Electrical brain stimulation has many therapeutic effects on neurological disorders. Brain stem and cerebellar stimulation have aided in some movement disorders; peroneal nerve stimulation used for treatment of dropfoot in stroke; dorsal column and deep brain stimulation used for treatment of chronic severe pain. Therapeutic application of brain stimulation lead to development of various new technologies of neurostimulation like transcranial magnetic stimulation, cortical brain stimulation and deep brain stimulation (DBS). ${ }^{1,2}$

In the field of neurostimulation techniques, deep brain stimulation developed as sub-specialty of neurosurgery. Since 1940, untreatable neurological and psychiatric conditions are being treated under this specialty. In 1987, deep brain stimulation was developed by the team treating the patients having tremors and Parkinson's disease. The stereotactic apparatus conducted the emergence of a new neurosurgical specialty, stereotactic neurosurgery, within which the skills, equipment and knowledge were developed that would later enable DBS to emerge as a therapy.,

Deep Brain Stimulation is also used to treat tremor, rigidity and dyskinesia along with a number of movement disorders. DBS has been a successful therapy in the surrounding area of the subthalamic nucleus (STN) and also control the integral part of the basal ganglia, which control complex movements. DBS has proved an effective treatment modality for movement disorders. ${ }^{5}$

\section{WHAT IS DEEP BRAIN STIMULATION?}

DBS consist of implanted electrodes that provide continuous tiny electrical currents into deep structures of the brain. ${ }^{6}$ Deep brain stimulation (DBS) therapeutical procedure in 
which low intensity, electric impulses, are applied typically at or near a frequency of $130 \mathrm{~Hz}$, to critical sites in the brain through permanently embedded electrodes. These impulses are transiently activated nearby axons; the distant effects of stimulation are dependent on the function of the certain neurons stimulation. Difference in transmission of pathway network structures (e.g., altered beta-oscillation in Parkinson's disease) this variation in current flow leads to an improvement in the symptoms of disease. DBS is delivered in square-wave impulses of amplitude 1-5 $\mathrm{V}$ (or $0.5-10 \mathrm{~mA}$ ) and duration $30-450 \mu \mathrm{s}$, with the precise values being determined by the indication and by clinically guided optimization in the individual patient. ${ }^{7}$

Deep brain stimulation device consist of following parts:

1. The electrode.

2. The pulse generator.

3. The extension.

The electrode - It is a small tip shaped device that is placed deep into the deep region of diseased brain. Its surface consists of four metal pads that generate the electric pulse. (Figure:1) The electric pulse generated by electrode only stimulates the brain tissue within close range of electrode.

The pulse generator - Also known as stimulator. It is a small, box-shaped device that generates the electrical signals for the electrode. Usually The pulse generator is embedded under the skin of the patient's chest. It consists of a battery with a lifespan of two to seven years. The electrical patterns are generated at quite high frequencies in fluctuating pulses usually over 100 times/second.

The extension - Extension is composed of insulated cable that carries signals from pulse generator to electrode implanted in the brain. To reduce the risk of infection from device through skin, surgeons use to make small path under skin from pulse generator to the electrode. ${ }^{8}$

Types of Deep Brain Stimulation Systems

There are three types of deep brain stimulators:

\section{Activa PC ${ }^{\mathrm{TM}}$ Neurostimulator}

The Activa ${ }^{\mathrm{TM}} \mathrm{PC}$ neurostimulator is a dual-channel device capable of delivering bilateral stimulation with a single device. Activa PC contains a non-rechargeable battery and microelectronic circuitry to deliver a controlled electrical pulse to the precisely targeted areas of the brain. (Figure:3)

\section{Activa RC Neurostimulator}

It is the first rechargeable and thinnest deep brain stimulation device available from Medtronic today. It has 9year lifespan. This dual-channel device act as a bilateral stimulator. (Figure:4)

\section{Activa SC Neurostimulator}

The Activa SC neurostimulator is a single-channel device with a daily maintenance-free battery that provides predictable longevity. It is the smallest nonrechargeable device in the Activa portfolio or deep brain stimulation from Medtronic. $^{9}$

\section{Types of deep brain stimulation}

Based upon target site in the brain there are three types of deep brain stimulation:
- The Thalamus (known as thalamic stimulation).

- The Globus pallidus (known as pallidal stimulation).

- The subthalamic nucleus (known as subthalamic stimulation).

\section{Thalamic deep brain stimulation}

In Thalamic deep brain stimulation, the DBS electrode is placed down into the thalamus and testing is started then Electrical impulses are transmitted from the tip of the electrode into the thalamus to locate a site of brain location where the tremor can be stopped effectively.

At the same time monitoring is done by team of the surgeon for any side effects of stimulation like numbness of the face, mouth, heaviness or weakness of the limb, change in speech, etc. If results are satisfactory the electrode is left in place and joined to a plastic clip that is attached to the skull opening and then wound is closed.

In second stage, a small incision is given below the collarbone to create a small pouch underneath the skin that will hold the pulse generator or battery.

Again a small incision is given behind the ear and a cable passed from the chest incision up to the head and then this cable is connected to the electrode coming out of the brain. All the entire system remains underneath the skin.

Subthalamic Deep Brain Stimulation - After creation of skull opening, a microelectrode (very small metal wire) is inserted into the brain towards the thalamus and subthalamic region. Identification of specific brain cells in these regions is done. The reason to map out the area for optimized placement of the electrode, during this time period the room is kept dark. The patient must be made comfortable as the brain is assessed. When the particular area is identified, test stimulation is performed to check that the electrode is in safe position that will not disrupt the function of brain. After that the electrode is placed in safe area and clipped to the skull bone-fastening device.

When surgical intervention on both sides of the brain is to be the done on at the same setting a second incision is made on the other side and the procedure is repeated.

In first stage of the surgery the electrodes are placed into the brain in one day. The scalp incisions are closed and the patient is asked to return 3-7 days later for the second stage of the procedure.

Second stage is also performed under general anesthesia. Cables and batteries are inserted into the neck and chest area. Once the device is inserted, neurologist activates the stimulators.

Globus Pallidus Deep Brain Stimulation - Exceptionally the procedure is similar to thalamic and subthalmic stimulation. The electrodes are placed into the brain using MRI stereotactic guidance in dystonia patient. Both electrode insertion are done under local anesthesia, cable and pulse generator placement is done under general anesthesia on the same day. ${ }^{5}$

\section{INDICATIONS}

Deep brain stimulation is indicated in treatment of motor symptoms of Parkinson's disease (PD), essential tremor, dystonia, and wide variety of neurological and psychiatric 
conditions including epilepsy, obsessive-compulsive disorder $(\mathrm{OCD})$, and major depression. Deep brain along with its target areas in brain are listed in Table $1 .{ }^{10}$

\section{EFFECTIVENESS OF DBS}

\section{Treatment-Resistant Depression (TRD)}

The first target investigated for DBS for treatment-resistant depression(TRD) was the subcallosal cingulate (SCC) white matter, occasionally referred to as $\mathrm{Cg} 25$ or Brodmann area 25.40. This target was chosen based on a neuro-imaging database which suggested that this region was critical for depression and the antidepressant response especially in TRD. ${ }^{11,12}$

Puigdemont d et al in 2015 conducted a study to confirm the efficacy and measure the impact of discontinuation of the electrical stimulation. He conducted a 6 -month double-blind, randomized, sham-controlled crossover study in implanted patients with previous severe treatment-resistant depression (TRD) experienced full recurrence due to long term stimulation. After more than 3 months of stable remission, patients were randomly assigned to two types of treatment arms: first is the ON-OFF arm and second is the OFF-ON arm. The ON-OFF arm, which involved active electrode stimulation for 3 months followed by sham stimulation for 3 months, and the OFF-ON arm, which involved sham stimulation for 3 months followed by active stimulation for 3 months. He concluded that DBS of the (subcallosal cingulate gyrus) SCG is an effective and safe treatment for severe forms of TRD and that continuous electrical stimulation is required to maintain therapeutic effects. ${ }^{13}$

An expanded study of 20 patients showed a $60 \%$ response rate at 1 year and a remission rate around 50\%-results that were generally maintained over several years. Of note, most symptoms of depression improved, with insomnia, decreased energy, interest and psychomotor speed, disturbed social contact, apathy, anhedonia, poor concentration, and planning, all showing improvement after 3, 6, and 12 months of follow-up. A more recent DBS target for TRD is the medial forebrain bundle (MFB), which includes ascending and descending white matter fibers connecting the ventral tegmental area with the nucleus accumbens. ${ }^{12,14,15,16}$

Other targets considered for DBS for TRD include: Inferior thalamic peduncle-this target may also have benefits for OCD, lateral habenular complex and rostral cingulate gyrus. ${ }^{17,18,19,20,21}$

\section{Tourette syndrome}

Tourette syndrome is defined by the presence of motor and vocal tics-rapid, repetitive, stereotyped movements or vocalizations-that manifest before the age of 18 years and last for more than 12 months in the absence of secondary causes. $^{22}$

Recently, nine targets are followed in DBS for TS, including the thalamic centromedian parafascicular complex (CMPf), the cross point of the centromedian nucleus-substantia periventricularis-nucleus ventro-oralis nucleus $\left(\mathrm{CM}-\mathrm{Spv}^{-}\right.$ $\mathrm{Voi}$ ), the target of the nucleus ventro-oralis posterior-ventro oralis anterior-Voi complex (Vop-Voa-Voi), the globus pallidus internus $(\mathrm{GPi})$ (anteromedial and posteroventral regions), the nucleus accumbens (NA), the anterior limb of the internal capsule (ALIC), the subthalamic nucleus (STN) and the globus pallidus externus $(\mathrm{GPe})^{23}$

In a recent study, three patients had electrodes placed in both the thalamus and the anteromedial $\mathrm{GPi}$, stimulation resulting in a YGTSS improvement of $65 \%-96 \%$ for amGPi, 30\%-64\% for CMPf and 43\%-76\% in combination stimulation. Follow up for as long as 60 months in 1 patient demonstrated maintenance of tic improvement. Pallidal stimulation showed to be more effective for tic reduction than thalamic or even combination stimulation. ${ }^{24}$

Savica R recently evaluated the efficacy of DBS in Tourette syndrome in 3 patients targeting the bilateral thalamic centromedian/parafascicular complex (CM/Pf). He found excellent outcome. At 1-year follow-up, the mean reduction in the total Yale Global Tic Severity Scale score in the 3 patients was 70\% (range, 60\%-80\%). ${ }^{25}$

\section{Anorexia Nervosa}

The anterior cingulated (AC), and in particular the subcallosal cingulate (SCC), appear to be implicated both structurally and functionally in the symptoms of AN. The $\mathrm{AC}$ is also involved in both mood states and the regulation of affect, both directly and through its influence on higher cortical structures. Finally, the ventral striatum, or nucleus accumbens (NAC), may be involved in reward circuits. ${ }^{26}$

Lipsman $\mathrm{N}$ et al evaluated the safety of DBS to modulate the activity of limbic circuits and effectiveness over clinical features of anorexia nervosa. preoperatively patients underwent medical optimisation and had baseline body-mass index (BMI), psychometric, and neuroimaging investigations, followed by embeded electrodes and pulse generators for continuous delivery of electrical stimulation. Patients were kept on follow up for 9 months after DBS activation, and the primary outcome of adverse events associated with surgery or stimulation was monitored at every follow-up visit. DBS was associated with improvements in mood, anxiety, affective regulation, and anorexia nervosa-related obsessions and compulsions in four patients and with improvements in quality of life in three patients after 6 months of stimulation. ${ }^{27}$

\section{Obsessive-Compulsive Disorders}

Two targets are covered in majority of cases as per literature review, the majority of published studies covered two targets: the subgenual cingulate gyrus ( $\mathrm{sgCC}$ or $\mathrm{Cg} 25)$ and the ventral internal capsule/ventral striatum (VC/VS). The latter was originally termed anterior limb of internal capsule (ALIC), but the name evolved as the target itself moved more posteriorly. OCD was the first psychiatric disorder targeted with DBS, following the theory that DBS creates a "virtual lesion" of the stimulated tissue. ${ }^{28}$

Alonso $\mathrm{P}$ et al determined the efficacy and tolerability of DBS in OCD and the

existence of clinical predictors of response using metaanalysis. Literature was searched on DBS for OCD from 1999 through January 2014 using PubMed/MEDLINE and PsycINFO. Fixed and random-effect meta-analysis was performed with score changes (pre-post DBS) on the YaleBrown Obsessive Compulsive Scale as the primary-outcome measure, and the number of responders to treatment, quality of life and acceptability as secondary measures. 31 studies 


\begin{tabular}{|l|l|l|}
\hline S. No. & Indications & Target \\
\hline 1 & Epilepsy & $\begin{array}{l}\text { Anterior thalamic nucleus, Cerebellum, caudate nucleus, subthalamic nucleus, } \\
\text { centromedian nucleus of the thalamus, locus coeruleus, mammillary bodies; } \\
\text { hippocampus }\end{array}$ \\
\hline 2 & Essential tremor & Ventral intermediate nucleus of the thalamus, subthalamic nucleus \\
\hline 3 & Parkinson's disease & Globus pallidus internus, subthalamic nucleus, pedunculopontine nucleus \\
\hline 4 & Primary dystonia & Globus pallidus internus, subthalamic nucleus \\
\hline 5 & Addiction & Nucleus accumbens, subthalamic nucleus \\
\hline 6 & Alzheimer's dementia & Nucleus basalis of Meynert, fornix \\
\hline 7 & Anorexia & Cingulate area 25 or subgenual cingulated \\
\hline 8 & Chorea & Globus pallidus internus \\
\hline 9 & Chronic pain & Periaqueductal gray; Ventral posterolateral/posteromedial thalamus \\
\hline 10 & Cluster headache & Posterior hypothalamus \\
\hline 11 & Depression & Cingulate area 25 Cg25, ALIC, NAc \\
\hline 12 & Holmes tremor & Vim, STN \\
\hline 13 & Impairment of Consciousness & CT \\
\hline 14 & Obesity & VMH, LH \\
\hline 15 & Schizophrenia & NAc/VS, VTA \\
\hline 16 & Tinnitus & LC \\
\hline 17 & Tourette's syndrome & CM thalamus, GPi, ALIC, NAc \\
\hline 18 & Obsessive compulsive Disorder & VC/VS, (ALIC, NAc, STN, ITP) \\
\hline & & Table-1: Diseases and target areas in the brain \\
\hline
\end{tabular}

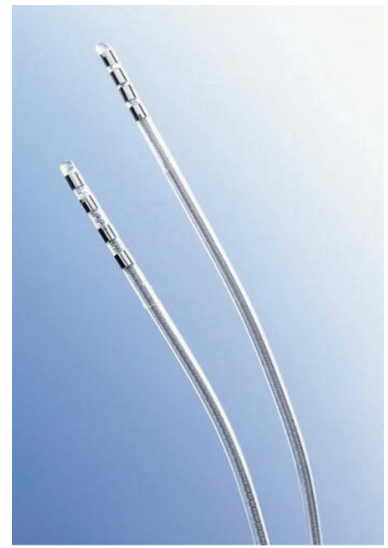

Figure-1: The Electrode

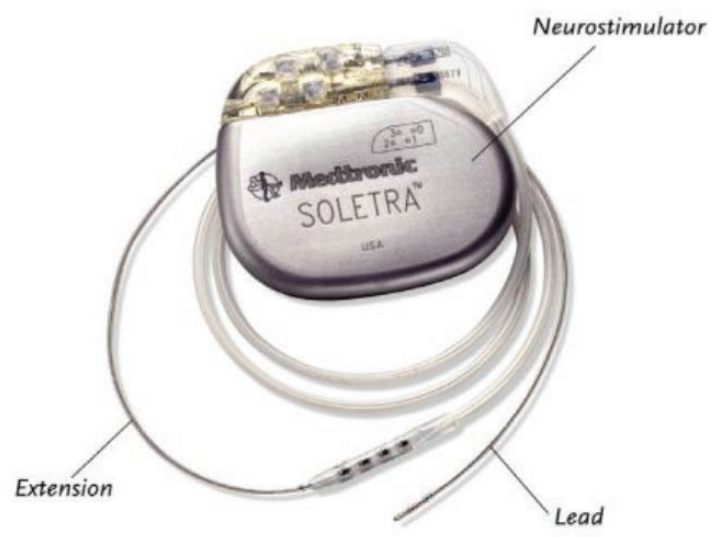

Figure-2: Pulse Generator

involving 116 subjects were identified. 83 subjects were implanted in striatal areas-anterior limb of the internal capsule, ventral capsule and ventral striatum, nucleus accumbens and ventral caudate -27 in the subthalamic nucleus and 6 in the inferior thalamic peduncle. He

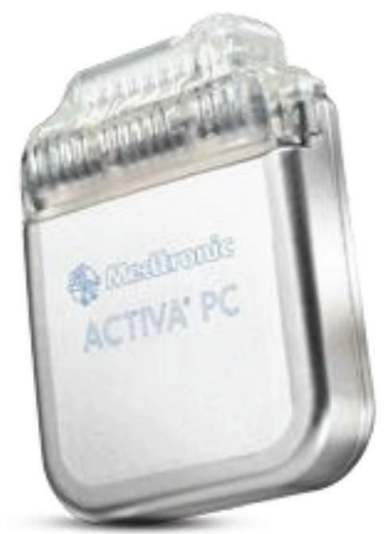

Figure-3: Activa PCTM Neurostimulator

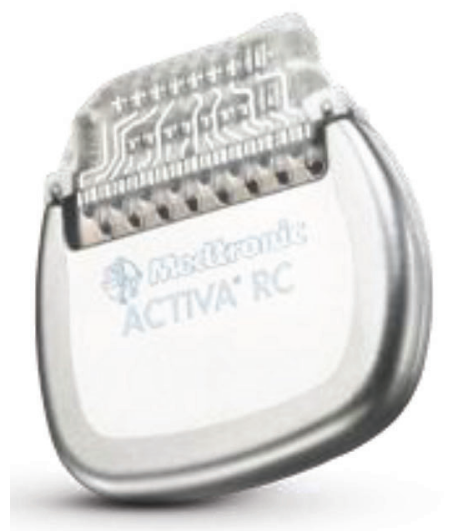

Figure-4: Activa RC Neurostimulator

confirmed that DBS constitutes a valid alternative to lesional surgery for severe, therapy-refractory OCD patients. ${ }^{29}$

\section{Obesity}

Obesity is a long term progressive disorder associated with 


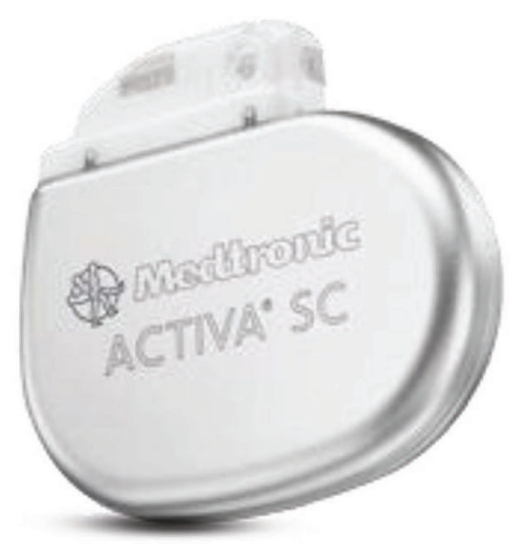

Figure-5: Activa SC Neurostimulator

a unhealthy life style and genetic disorder with prevalence of 600 million individuals Worldwide. ${ }^{30}$ DBS targets currently proposed to treat obesity include the hypothalamus and nucleus accumbens (NAc). The hypothalamus may be subdivided in various anatomical and functional subregions/ nuclei. Some of the most commonly involved in mechanisms of feeding and energy balance are the arcuate nucleus (ARC), the dorsal medial nucleus, the paraventricular nucleus, the lateral hypothalamus $(\mathrm{LH})$ and the ventral medial nucleus $(\mathrm{VM})$. $^{31,32}$

The VM is a relatively large nucleus with an abundance of leptin receptors. Leptin and insulin provide the hypothalamus with peripheral signals of adiposity. The organism reduces feeding when the harmones leval are high. In the hypothalamus, prominent levels of leptin and insulin receptors also be found in ARC. ${ }^{31,32,33}$ In this nucleus, these hormones modulate activity of populations of cells expressing neuropeptide Y/agouti gene-related protein and cocaine- and amphetamine-related transcript (CART)/ pro-opiomelanocortin (POMC). In general, states of negative energy deficit (e.g., starvation) increase the activity of ARC NPY/AGRP neurons, ultimately favoring food consumption. Overall, ARC NPY/AGRP neurons project to most hypothalamic nuclei involved in feeding control. The LH extends through most of the anteropoesterior axis of the hypothalamus subpopulations of cells in LH express different peptides and hormones, including orexins and melanin-concentrating hormone (MCH). These harmones have orexigenic effects and increases the rate of food consumption. ${ }^{31,34,35}$

\section{Addiction and Substance Use Disorder}

Commonly referenced addictions include substance abuse, gambling, and even

Internet gaming, all of which are now listed as disorders in the 5th edition of the Diagnostic and Statistical Manual of Mental Disorders. ${ }^{36}$

Neuro-imaging studies in humans have identified broad alterations in prefrontal, limbic, and cortical areas that are involved in addiction and maladaptive behavior but the most consistent core regions appear to be involved in the dopaminergic connection between the VTA and the NAcc, which modulates learning, memory, and repetitive behaviors. $^{37}$
Voges J et al treated 5 patients off-label with bilateral NAc DBS for severe alcohol addiction, all patients' experienced significant and ongoing improvement of craving. Two patients remained completely abstinent for more than 4 years. ${ }^{38}$

Henderson $\mathrm{MB}$ et al conducted a study to evaluate that DBS is effective in reducing both alcohol preference and $\mathrm{ADE}$ in rats known to prefer alcohol with no sucrose fading or any other behavioral modification to induce alcohol consumption. It was concluded that NAcc plays a key role in the rewarding and subsequent addictive properties of drugs of abuse in general and of alcohol in particular. Moreover, DBS coupled with other neurophysiological measurements may be a useful tool in determining the role of the NAcc in the mesocorticolimbic reward circuit. ${ }^{39}$

Similarly, in heroin addicted patients NAc, DBS induced sustained abstinence reduced craving, and was accompanied as well by a decrease in the number of cigarettes smoked. ${ }^{40}$

\section{PARKINSON'S DISEASE}

Deep brain stimulation (DBS) of the subthalamic nucleus $(\mathrm{STN})$ and the internal globuspallidus (GPi) or ventral intermedius nucleus (VIM) of the thalamus has developed and become a distinguished symptomatic treatment for Parkinson's disease (PD). Especially DBS of the STN and $\mathrm{GPi}$ is an effective option to improve motor symptoms and manage long-term motor complications resulting from levodopa treatment, such as wearing-off phenomena and dyskinesias. Furthermore patients' mobility, activities of daily living, emotional well-being and health-related quality of life which are impaired by motor symptoms and complications can be enhanced by DBS. ${ }^{41}$

In contrast to STN- and GPi-DBS, stimulation of the VIM has no effects on dyskinesia, motor fluctuations, rigidity and bradykinesia but a clear and immediate effect on tremor. Certainly, VIM-DBS is a therapeutic option for patients with essential tremor and elderly patients with a unilateral tremor-dominant PD. Another target point is the pedunculopontine nucleus (PPN). DBS of the PPN has been analyzed in small experimental trials. There is evidence revealing that stimulation of the PPN might have positive effects on parkinsonian gait disorder, postural instability and freezing. ${ }^{41}$

\section{ADVERSE EVENTS}

The DBS System may either get affected by or may adversely affect by various medical equipment such as cardioverter/ defibrillators, cardiac pacemakers, external defibrillators, ultrasonic equipment, electrocautery, or radiation therapy. MRI conditions causes excessive heating at the lead electrodes which can causes a serious injury, such as coma, paralysis, or death, or that may cause device damage.

Adverse effects related to the therapy, device can include intracranial hemorrhage, cerebral infarction, CSF leak, pneumocephalus, seizures, surgical site complications (including pain, infection, dehiscence, erosion, seroma, and hematoma), meningitis, encephalitis, brain abscess, cerebral edema, aseptic cyst formation, device complications. ${ }^{9}$ 
Deep Brain Stimulation Therapy in India

Cities/State in India that offers Deep Brain Stimulation Therapy at best hospitals are as follows;

\begin{tabular}{|l|l|l|}
\hline Mumbai & Hyderabad & Kerala \\
\hline Delhi & Pune & Goa \\
\hline Bangalore & Nagpur & Jaipur \\
\hline Chennai & Gurgaon & Chandigarh \\
\hline
\end{tabular}

\section{Cost of Deep Brain Stimulation Therapy in India}

Deep Brain Stimulation Therapy in India is highly economical. The total price can be $60-90 \%$ cheaper than in western country. The final price includes medicine cost, doctor's fee, consultation fee, nursing, pre and post accommodation at hotels, private transportation, patient economical status etc. The cost of deep brain stimulation varies. The amount mentioned below includes the system and the surgery itself, along with inpatient stay and follow-up assessments.,

\begin{tabular}{|l|c|c|}
\hline India & US & UK \\
\hline$\$ 15,000-30,000$ & $\$ 60,000-\$ 70,000$ & $\$ 55,000-\$ 65,000$ \\
\hline
\end{tabular}

\section{FDA APPROVAL}

In 1997, the Activa ${ }^{\circledR}$ Tremor Control System (Medtronic) was cleared for marketing by the U.S. Food and Drug Administration (FDA) for deep brain stimulation. The original FDA-labeled indications were limited to unilateral implantation of the device for the treatment of tremor, but, in January 2002, the FDA-labeled indications were expanded to include bilateral implantation as a treatment to decrease the symptoms of advanced Parkinson disease that are not controlled by medication. In April 2003, the labeled indications were further expanded to include “...unilateral or bilateral stimulation of the internal globus pallidus or subthalamic nucleus to aid in the management of chronic, intractable (drug refractory) primary dystonia, including generalized and/or segmental dystonia, hemidystonia, and cervical dystonia (torticollis) in patients seven years of age or above." ${ }^{42}$

\section{CONCLUSION}

Deep brain stimulation is now a days used in specialized centers and with the specialist to treat diseases that are unmanageable to medical treatment. It has been approved in Europe for the treatment of movement disorders (Parkinson's disease, tremor, dystonia), medically intractable epilepsy, and obsessive-compulsive disorder. In view of its clinical efficacy of DBS has already been established and it has been recommended in various medical publications, especially for the treatment of movement disorders, the underutilization of this technique by general practitioners and specialists (particularly those in private practice) is surprising. The success of DBS depends largely on a wellfunctioning interdisciplinary team. There is also a need for appropriate training of the physicians who will care for these patients over the long term, in order to raise their competence and self-confidence

\section{REFERENCES}

1. Electrical Stimulation of the Brain (ESB) [homepage on the internet]. No date [cited on 2018 Mar 19].
2. Schlaepfer TE, Saviane C. The development of deep Brain stimulation for Neurological and Psychiatric disorders: Clinical, societal and Ethical issues. Frontiers in Integrative Neuroscience; 2012 [cited on 2018 Mar 19].

3. Hariz ML, Blomstedt P, Zrinzo L. Deep brain stimulation between 1947 and 1987: The untold story. Neurosurg Focus. 2010; 29(2):E1.

4. Spiegel EA, Wycis HT, Marks M, Lee AJ. Stereotaxic apparatus for operations on the human brain. Science. 1947;106(2754):349-50.

5. Bojwani D. Deep brain stimulation in India. c2013 [cited on 2018 Mar 19].

6. Ford B. Treating Parkinson's Deep Brain Stimulation. Parkinson's Disease Foundation. c2016 [cited on 2018 Mar 20].

7. Coenen VA ${ }^{1}$, Amtage F, Volkmann J, Schläpfer TE. Deep Brain Stimulation in Neurological and Psychiatric Disorders. Dtsch Arztebl Int. 2015;112(31-32):519-26.

8. Clements IP. How deep brain stimulation work. c2018 [cited on 2018 Mar 19].

9. Deep brain stimulation systems, health care professionals [homepage on the internet]. c2018 [cited on $2018 \mathrm{Mar}$ 19].

10. Herrington TM, Cheng JJ, Eskandar EN. Eskandar Mechanisms of deep brain stimulation. J Neurophysiol. 2016; 115(1):19-38.

11. Mayberg HS, Lozano AM, Voon V, McNeely HE, Seminowicz D, Hamani C et al. Deep brain stimulation for treatment-resistant depression. Neuron. 2005; 45(3):651-60.

12. Mayberg HS. Targeted electrode-based modulation of neural circuits for depression. J Clin Invest. 2009; 119(4):717-25.

13. Puigdemont D, Portella M, Pérez-Egea R, Molet J, Gironell A, de Diego-Adeliño J et al. A randomized double-blind crossover trial of deep brain stimulation of the subcallosal cingulate gyrus in patients with treatment-resistant depression: a pilot study of relapse prevention. J Psychiatry Neurosci 2015;40(4) 224-31.

14. Lozano AM, Mayberg HS, Giacobbe P, Hamani C, Craddock RC, Kennedy SH. Subcallosal cingulate gyrus deep brain stimulation for treatment-resistant depression. Biol Psychiatry. 2008;64(6):461-7.

15. Kennedy SH, Giacobbe P, Rizvi SJ, Placenza FM, Nishikawa Y, Mayberg HS et al. Deep brain stimulation for treatment-resistant depression: follow-up after 3 to 6 years. Am J Psychiatry. 2011;168(5):502-10.

16. Coenen VA, Panksepp J, Hurwitz TA, Urbach H, Madler B. Human medial forebrain bundle (MFB) and anterior thalamic radiation (ATR): imaging of two major subcortical pathways and the dynamic balance of opposite affects in understanding depression. J Neuropsychiatry Clin Neurosci. 2012; 24(3):223-36.

17. Jiménez F, Velasco F, Salin-Pascual R, Hernández JA, Velasco M, Criales JL et al. A patient with a resistant major depression disorder treated with deep brain stimulation in the inferior thalamic peduncle. Neurosurgery. 2005; 57(5):585-93.

18. Jiménez F, Velasco F, Salín-Pascual R, Velasco M, Nicolini H, Velasco AL et al. Neuromodulation of the 
inferior thalamic peduncle for major depression and obsessive compulsive disorder. Acta Neurochir Suppl. 2007; 97(Pt 2):393-8.

19. Jimenez F, Nicolini H, Lozano AM, Piedimonte F, Salin R, Velasco F. Electrical stimulation of the inferior thalamic peduncle in the treatment of major depression and obsessive compulsive disorders. World Neurosurg. 2013;80(3-4):S30.e17-25.

20. Sartorius A, Kiening KL, Kirsch P, von Gall CC, Haberkorn U, Unterberg AW et al. Remission of major depression under deep brain stimulation of the lateral habenula in a therapy-refractory patient. Biol Psychiatry. 2010; 67(5):e9-e11.

21. Sakas DE, Panourias IG. Rostral cingulate gyrus: A putative target for deep brain stimulation in treatmentrefractory depression. Med Hypotheses. 2006; 66(2):491-4.

22. Leckman JF, Bloch MH, Scahill L, King RA. Tourette syndrome: the self under siege. J Child Neurol. 2006; 21(8):642-9.

23. Fraint, $\mathrm{A}, \mathrm{Pal}, \mathrm{G}$. Deep brain stimulation in Tourette's syndrome. Front Neurol. 2015; 6(1):170.

24. Houeto JL, Karachi C, Mallet L, Pillon B, Yelnik $\mathrm{J}$, Mesnage $\mathrm{V}$ et al. Tourette's syndrome and deep brain stimulation. J. Neurol. Neurosurg. Psychiatry 2005;76(3):992-5.

25. Savica R, Stead M, Mack KJ, Lee KH, Klassen BT. Deep Brain Stimulation in Tourette Syndrome: A Description of 3 Patients With Excellent Outcome. Mayo Clin Proc. 2012; 87(1):59-62.

26. Woodside DB, Lipsman N, Lozano AM. Deep Brain Stimulation in Chronic and Severe Anorexia Nervosa. Psychiatric Times 2014 Mar 3 [cited on 2018 Mar 19].

27. Lipsman $\mathrm{N}^{1}$, Woodside DB, Giacobbe P, Hamani C, Carter JC, Norwood SJ, et al. Subcallosal cingulate deep brain stimulation for treatment-refractory anorexia nervosa: a phase 1 pilot trial. Lancet. 2013; 381(9875):1361-70.

28. Widge AS, Dougherty DD. Deep Brain Stimulation for Treatment-Refractory Mood and ObsessiveCompulsive Disorders. Curr Behav Neurosci Rep 2015; 2(1):187-97.

29. Alonso P, Cuadras D, Gabriëls L, Denys D, Goodman W, Greenberg BD et al. Deep Brain Stimulation for Obsessive- Compulsive Disorder: A Meta-Analysis of Treatment Outcome and Predictors of Response. PLoS One. 2015; 10(7):e0133591.

30. World Health Organization (WHO). Obesity and Overweight.c2018 [cited on 2018 Mar 19].

31. Williams G, Bing C, Cai XJ, Harrold JA, King PJ, Liu XH. The hypothalamus and the control of energy homeostasis: Different circuits, different purposes. Physiol. Behav. 2001; 74(6):683-701.

32. Simpson KA, Martin NM, Bloom SR. Hypothalamic regulation of food intake and clinical therapeutic applications. Arq. Bras. Endocrinol. Metabol. 2009; 53(4):120-8.

33. Elmquist JK, Elias CF, Saper CB. From lesions to leptin: Hypothalamic control of food intake and body weight. Neuron 1999; 22(3):221-32.

34. Elmquist JK. Hypothalamic pathways underlying the endocrine, autonomic, and behavioral effects of leptin. Physiol. Behav. 2001; 74(5): 703-8.

35. Stuber GD, Wise RA. Lateral hypothalamic circuits for feeding and reward. Nat. Neurosci. 2016; 19(2):198205.

36. Bradizza CM, Stasiewicz PR, Paas ND. Relapse to alcohol and drug use among individuals diagnosed with co-occurring mental health and substance use disorders: a review. Clin Psychol Rev 2006; 26(1):162-78.

37. Koob GF, Volkow ND. Neurocircuitry of addiction. Neuropsychopharmacology 2010; 35(4):217-38.

38. Voges J, Müller U, Bogerts B, Münte T, Heinze HJ. Deep brain stimulation surgery for alcohol addiction. World Neurosurg. 2013; 80(3-4):S28.e21-31.

39. Henderson MB, Green AI, Bradford PS, Chau DT, Roberts DW, Leiter JC. Deep brain stimulation of the nucleus accumbens reduces alcohol intake in alcoholpreferring rats. Neurosurg Focus. 2010; 29(2):E12.

40. Zullino DF, Thorens G, Krack P.Deep Brain Stimulation as a Treatment for Addictions: Could Impulsivity be the Therapeutic Target? Acta Psychopathol. 2016, 2(1):39.

41. Moldovan AS, Groiss SJ, Elben S, Südmeyer M, Schnitzler A, Wojtecki L. The treatment of Parkinson's disease with deep brain stimulation: current issues. Neural Regen Res. 2015; 10(7): 1018-22.

42. Deep brain stimulation. Protocol [homepage on the internet]. 2015 Jan 7 [cited on 2018 Mar 19]

Source of Support: Nil; Conflict of Interest: None

Submitted: 01-07-2018; Accepted: 17-08-2018; Published online: 21-08-2018 